\title{
Digital TWIN AND VirTUAl SENSOR FOR A ROTOR SYSTEM
}

\author{
Tuomas Tiainen, Jesse Miettinen, Raine Viitala, Kari Hiekkanen, Petri Kuosmanen
}
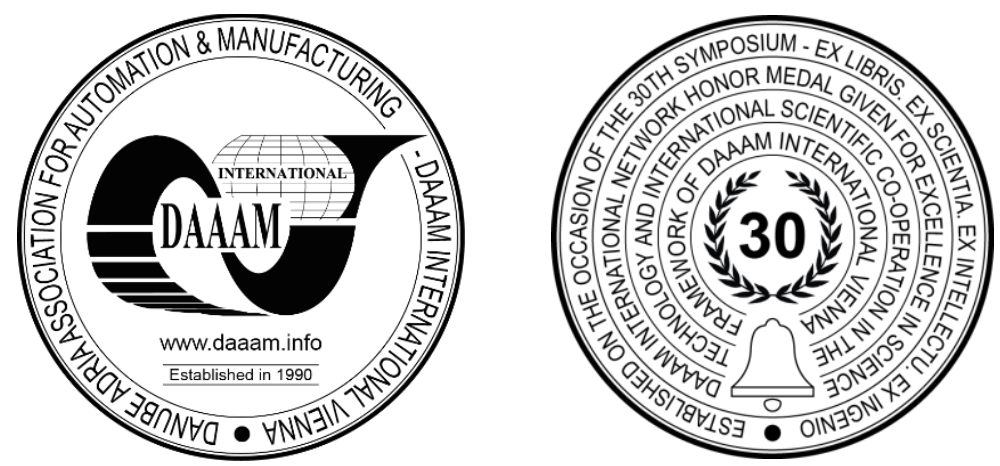

This Publication has to be referred as: Tiainen, T[uomas]; Miettinen, J[esse]; Viitala, R[aine]; Hiekkanen, K[ari] \& Kuosmanen, P[etri] (2019). Digital Twin and Virtual Sensor for a Rotor System, Proceedings of the 30th DAAAM International Symposium, pp.1115-1121, B. Katalinic (Ed.), Published by DAAAM International, ISBN 978-3-90273422-8, ISSN 1726-9679, Vienna, Austria

DOI: $10.2507 / 30$ th.daaam.proceedings.156

\begin{abstract}
A digital twin test bench was created to demonstrate the digital twin concept for control and prediction of the dynamic behavior of a paper machine roll. The paper presents a complete proof of concept digital twin system with wireless sensoring with flexible measurement patterns, data transfer, storage and visualization in an interactive 3D view. A virtual sensor based on a recurrent neural network was created to predict the middle cross section center point movement of a large flexible rotor based on acceleration and force input measured from bearing housings at the ends of the rotor. The results show that a neural network algorithm is feasible for predicting the dynamic behaviour of the rotor system. Future research at Aalto University aims to apply sensor fusion data as input to non-physics based models with the goal to predict key performance indicators of complex mechanical systems.
\end{abstract}

Keywords: digital twin; virtual sensor; rotor system

\section{Introduction}

The digital twin is a widely debated subject and several definitions exist for it. One definition of the digital twin is that the digital twin is the physical product and its virtual representation; including the linking data connecting the digital data from different sources to the physical product in question [1]. In this paper, the digital twin is defined as any virtual entity linked to a real world entity. 


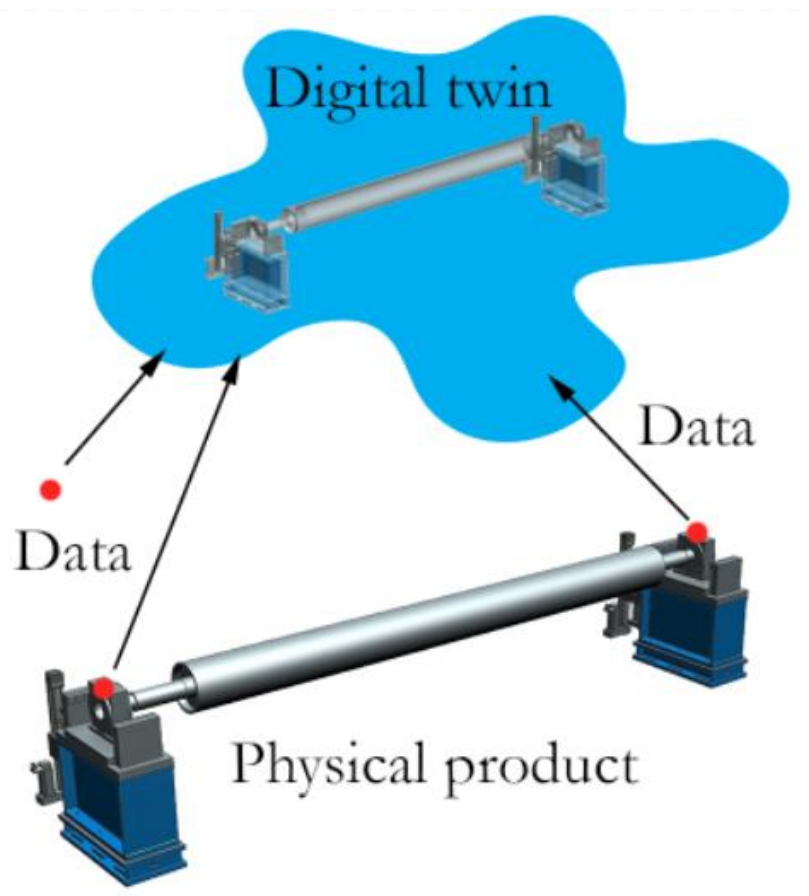

Fig. 1. The digital twin as a collection of information related to a physical product. The source of the information can be the physical product itself or a virtual source such as simulations and other virtual models.

Data from a product's lifecycle can be isolated, fragmented and stagnant, because there is no convergence between the physical product and its virtual representation [2]. Additionally, data can be split between process networks, with different parts of the network (such as manufacturing or maintenance) not having access to a product's data [3]. In other words, there is no product-centric view to the data from different stages of the product lifecycle. The concept of the digital twin aims to solve this problem by relating available data from a physical product into a holistic virtual representation of the product. The concept of product-centric data aggregation is not new [3], but recent advances in information technology have enabled applications that have not been viable previously. Declining costs for sensors, data transfer and storage and advanced analytics have enabled using big data to improve the design, manufacturing and operation of machines and other engineering products. Improvements in information technology have made the digital twin concept and many related applications feasible. Especially the ongoing miniaturization and price decline of data acquisition, transfer and storage technologies [4] are reasons for the increased benefits.

This paper presents a proof of concept of a digital twin featuring data collection, data aggregation from multiple sources, a user interface and a virtual sensor. The focus of this paper is to demonstrate a novel application of a recurrent neural network algorithm as a virtual sensor in a rotor system. More specifically, the aim of this study was to predict the dynamic behaviour of a large flexible rotor from sensor fusion data measured from bearing housings at the ends of the roll. In such research, the research questions are more philosophical in nature, and the interest is not only in how accurate the predictions of the algorithm are. Which variables in a rotor system have connections with each other? What does the neural network actually learn from the rotor system? Is simulation data suitable as training data for a neural network? Does the neural network generalize for other rotors or other operating conditions?

The scope of this research is limited to demonstrating the feasibility of predicting the dynamic behaviour of a rotor system with a neural network algorithm with data from two sensors in the bearing housings as input. Results based on measured data and a qualitative analysis of the model predictions are presented. In this application, the function of the neural network is, from the input data, to recognize patterns which are related to the predicted variable. Furthermore, similar methods could be applied to create any virtual sensors from any data, given that the data is available and contains aggregated information of some phenomenon.

\section{Proposed Digital Twin System}

The authors propose a digital twin system based on the definition that the digital twin links virtual data to a real world system [1,3]. Based on several identified features (Figure 2), a digital twin test bench was built at Aalto University ARotor laboratory. The authors propose and have created a digital twin system with the following features. 


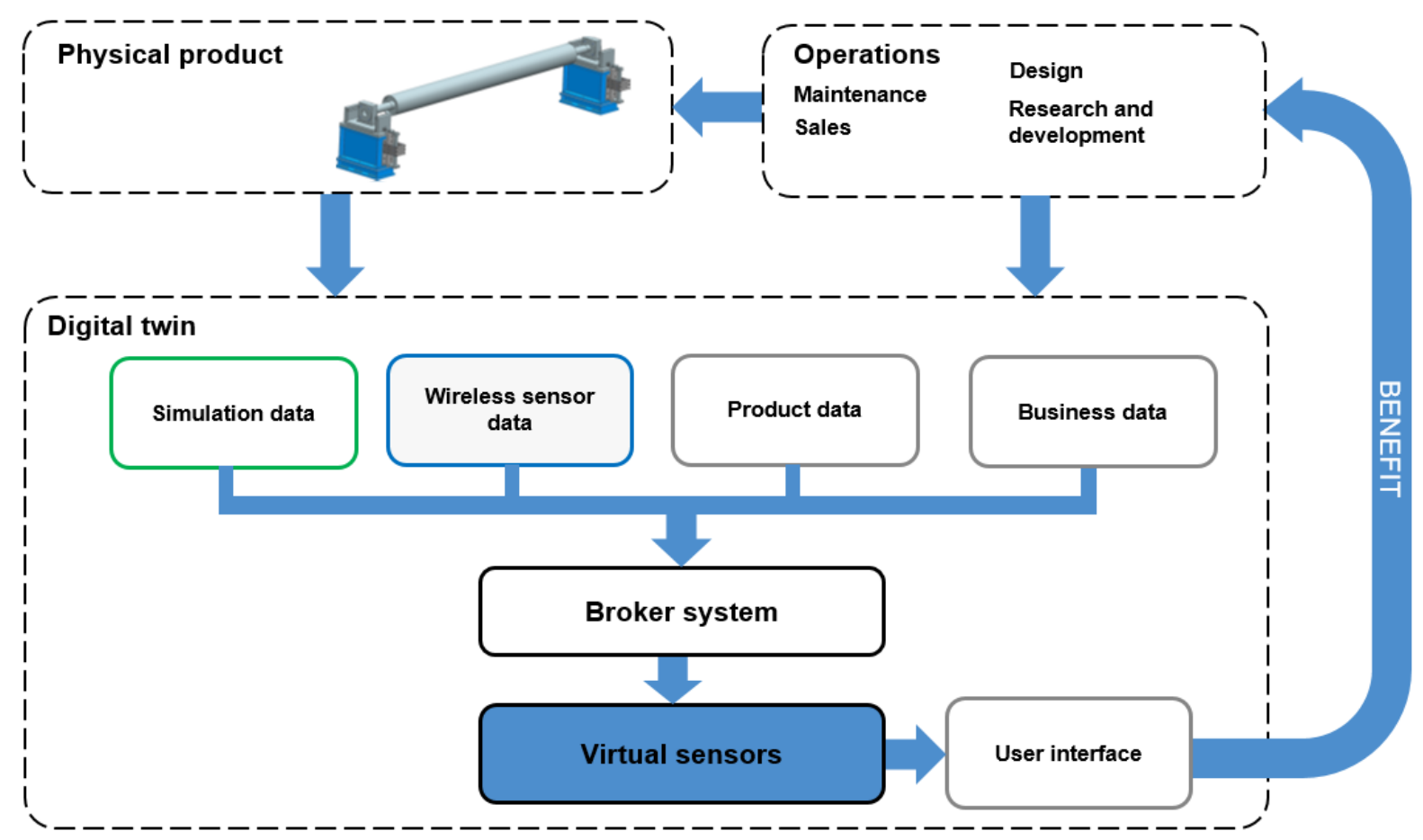

Fig. 2. Data flow and proposed features in the digital twin system

\section{Physical test bench}

The physical product in this study was a full-scale research paper machine roll research setup (Figure 3) at Aalto University ARotor laboratory. The system includes a device for adjustable foundation stiffness and a loading device to simulate real loading conditions.

The dynamic behaviour of large flexible rotors such paper machine rolls can have a direct effect on the quality of the end product. Research in the dynamics of large flexible rotors has been directed at subcritical vibrations, since large flexible rotors are typically operated below their critical speed (1st mode natural frequency). [5]

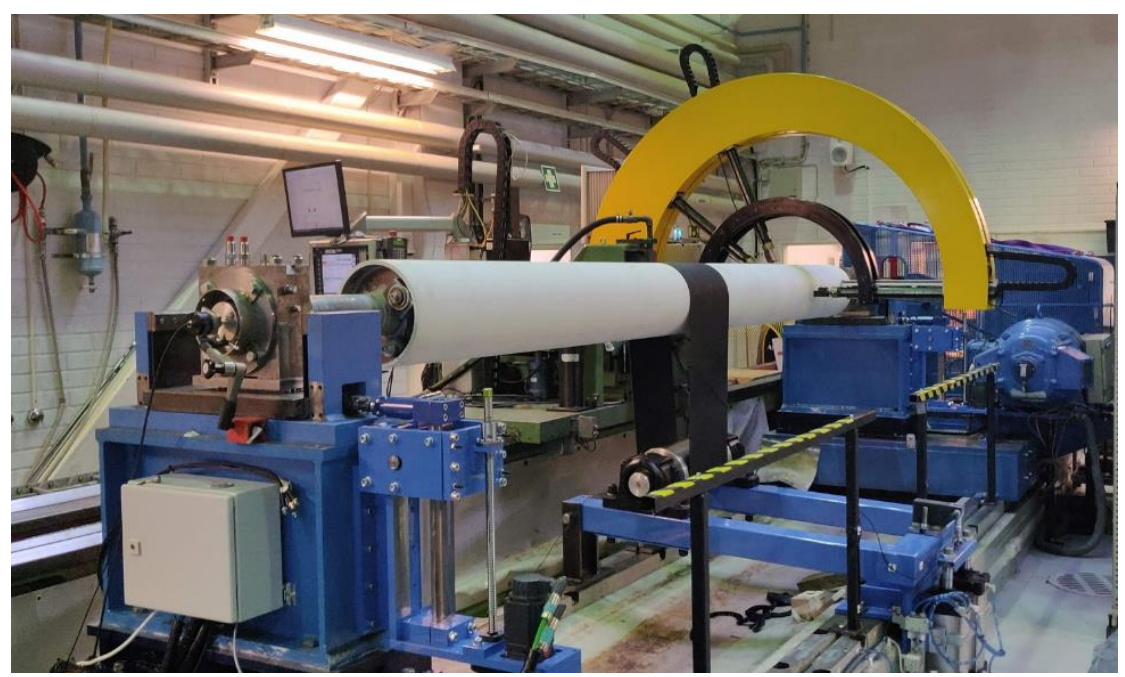

Fig. 3. The rotor test bench at ARotor laboratory 


\section{Sensoring}

The test bench incorporates versatile sensoring. For measurements of dynamic behaviour of the roll, the test bench has a four-point measurement frame with laser sensors. The dynamic behaviour of a large flexible rotor can be measured with multi-probe roundness measurement methods, since the center point movement of a rotating workpiece in the probe direction can be calculated by subtracting the roundness profile from the runout signal. [5]

The sensoring of the test bench also includes wireless accelerometers, which were not used in this research. Instead, more accurate wired force sensors and accelerometers were used. An accurate rotary encoder was attached to the end of the roll, which was used to trigger the measurement.

\section{Broker system}

A broker system was developed to aggregate data from different sources under a single product-centric view. The broker was implemented as a REST web service. The information model in the broker is based on the ODF Open Data Format standard [6]. In the broker, data is stored as infoitem objects under assets which receive a unique ID. Metadata related to infoitems can be used to filter the data. The broker can provide compound views of information related to a single asset. The sensors in the test bench integrate into the broker so that data related to the roll system can be viewed and filtered based on the broker endpoint address.

\section{User interface}

The system features a 3D user interface, which can be used to start and stop the roll rotation, to visualize sensor data and the analysis results. The 3D user interface uses endpoints from the broker as data sources for the visualizations and control features

\section{Virtual sensors}

The system features virtual sensors. A virtual sensor is a system predicting a variable, based on connections between other variables in the system, not direct measurement. In the present study, the virtual sensor was realized using a neural network combining sensor data from for a prediction of the middle cross section center point movement of the roll.

\section{Methods}

Neural networks (NNs) are computational models capable of learning underlying structures from data. All NNs are made of multiple layers of nodes with nonlinear connections between layers [7]. Recurrent Neural Networks (RNN) are neural networks that are relatively efficient at learning structures from sequential data [7, 8]. A state-of-the-art RNN model is the Long Short-Term Memory (LSTM) network, which has functions not only for remembering long-term and short-term phenomena but also forgetting some earlier observed phenomena. These functions have been showcased for example in text prediction [9].

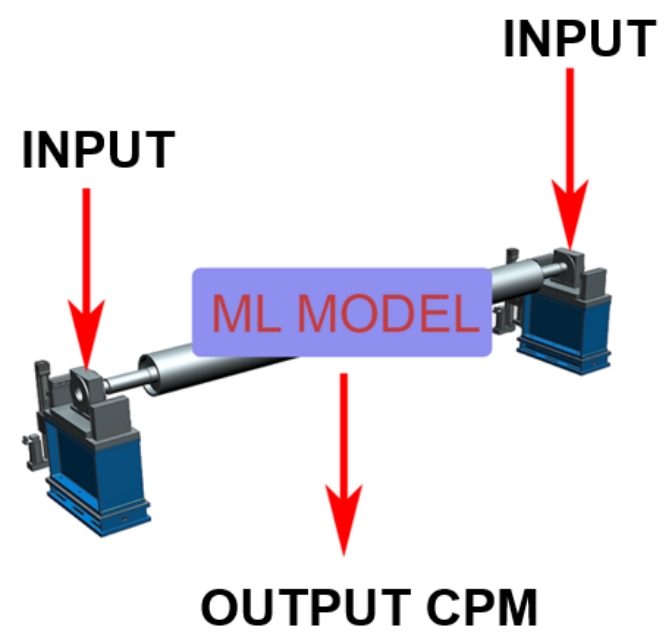

Fig. 4. A recurrent neural network algorithm was trained to predict middle cross section center point movement of the roll based on force or acceleration input data measured from bearing housings at the ends of the roll.

A virtual sensor based on LSTM was created to predict the rotor center point cross section movement based on force and acceleration sensor measurements from bearing housings at the ends of the rotor (Figure 4). The data used in these measurements was a time series of 2763 samples, which results in time windows of 1.4 seconds with $2000 \mathrm{~Hz}$ sampling rate. A supervised learning approach was used in the training of this virtual sensor. A random search was conducted to 
optimize the neural network architecture (hyperparameters). Searched parameters included hidden size, number of layers, drop-out rate, batch size, window length and time steps between window starts.

A signal consisting of discrete time steps $S_{n}$ was sliced into time windows (Figure 5 a). Each input signal was windowed so that the neural network will see an even distribution of start and end conditions for the rotor system.

Figure $5 \mathrm{~b}$ shows the structure of the inputs and output of the neural network. The input matrix on the left contains force or acceleration data and the output matrix on the right contains the center point movement coordinates. $\mathrm{N}$ samples of two coordinate pairs as input produce $\mathrm{N}$ samples of center point movement coordinate pairs as output. As shown in Figure $5 \mathrm{~b}$, each time window has $\mathrm{N}$ discrete time steps consisting, for each time step, of two pairs of vertical and horizontal sensor measurement values both ends as the input part. Each time step $S_{n}$ has two values for the middle cross sections center position movement coordinates as the target part.

During training and testing, the input part of the time window was given for the LSTM -network and the outputs were compared with the mean squared error (MSE) function to the target part of the whole time window. Thus, the LSTMnetwork was used as a sequence-to-sequence predictor of time series data.

a)

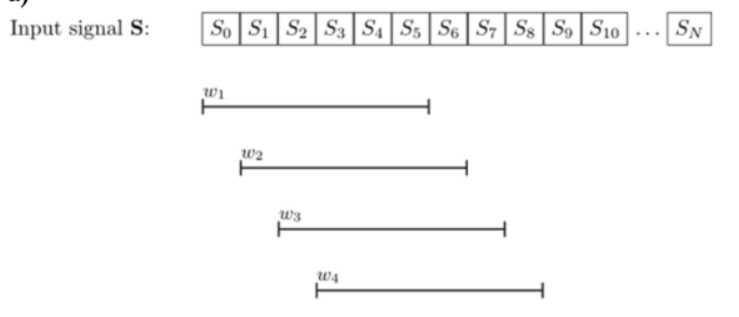

b)

$$
\left[\begin{array}{cccc}
x_{R 1} & y_{R 1} & x_{L 1} & y_{L 1} \\
x_{R 2} & y_{R 2} & x_{L 2} & y_{L 2} \\
\vdots & & & \\
x_{R N} & y_{R N} & x_{L N} & y_{L N}
\end{array}\right] \Longrightarrow\left[\begin{array}{cc}
x_{C 1} & y_{C 1} \\
x_{C 1} & y_{C 1} \\
\vdots & \\
x_{C N} & y_{C N}
\end{array}\right]
$$

Fig. 5. Structuring of data in the neural network.

Several datasets were obtained for experiments. A simulation dataset of 12000 similar rolls with randomly varied rotating speed, horizontal support stiffness, density and bearing clearance was generated. In the simulations, 9 seconds of samples with $2000 \mathrm{~Hz}$ sampling rate were captured from 12000 simulated cases.

For the measured data, force and acceleration values and displacement data from four laser probes was measured between $4 \mathrm{~Hz}-18 \mathrm{~Hz}$ rotational speeds. Each measured sample was triggered by the rotary encoder attached to the roll, 1024 values for each rotation. The data was resampled to $2000 \mathrm{~Hz}$, which was possible since the sampling triggered by the rotary encoder with 1024 pulses per round and $4 \mathrm{~Hz}$ rotational frequency (which was the lowest rotational frequency used in the measurements) of the roll accounts for a sampling rate of over $4 \mathrm{kHz}$. The data was used to calculate the center point movement of the measured cross section with the redundant diameter four-point roundness measurement algorithm.

To answer the research questions, four different experiments were designed:

1. In the first experiment, only data sampled from simulation was used for training and testing.

2. In the second experiment, only data sampled from the real system was used for training and testing.

3. In the third experiment, data sampled from the simulation was planned to be used in training and data sampled from the real system was planned to be used in testing.

4. The fourth experiment was supposed to conclude training with mixed data from simulation and real system and testing with data from the measurements.

The third and fourth experiments were to be conducted if both first and second experiments would show success.

\section{Results}

A preliminary test with the neural network predicting runout in the center based on a runout signal from the end, training with low rotational frequencies $(4 \mathrm{~Hz}-7.5 \mathrm{~Hz})$ and predicting the higher rotational frequencies $(7.5 \mathrm{~Hz}-18$ $\mathrm{Hz}$ ). Based on the promising preliminary results, the research was focused on predicting actual center point movement.

In the first experiment with simulation data, the neural network learned to predict sequences of multiple seconds with MSE of less than 0.01 in the first experiment. However, in this case the network was only trained and tested with sequences taken from an individual simulation. Additionally, a problem with the data from the simulations was found 
after some analytical observations. The simulations were noticed to produce unrealistic vibrational patterns and the simulation was thus deemed unsuitable for the experiment.

The research was then focused on experiments with real measured data from the rotor system. After parameter optimisation the virtual sensor learned to predict sequences of center point movement from force sensor data. The neural network was tested on data which it had never seen before sampled from the real system from a speed range of $10 \mathrm{~Hz}-$ $18 \mathrm{~Hz}$. Convergence of the training was not achieved with accelerometer data. It was assumed that the input data from accelerometers had too much noise. However, force sensors were accurate enough for the training to converge. Ultimately, the virtual sensor was able to predict sequences of middle cross section center point movement with MSE of less than 0.01. One example of such successful predictions is shown in Figure 6.

The third and fourth experiments with a network trained with simulation data predicting from measured data were not performed since the data from the simulation did not seem to represent the real system sufficiently well.
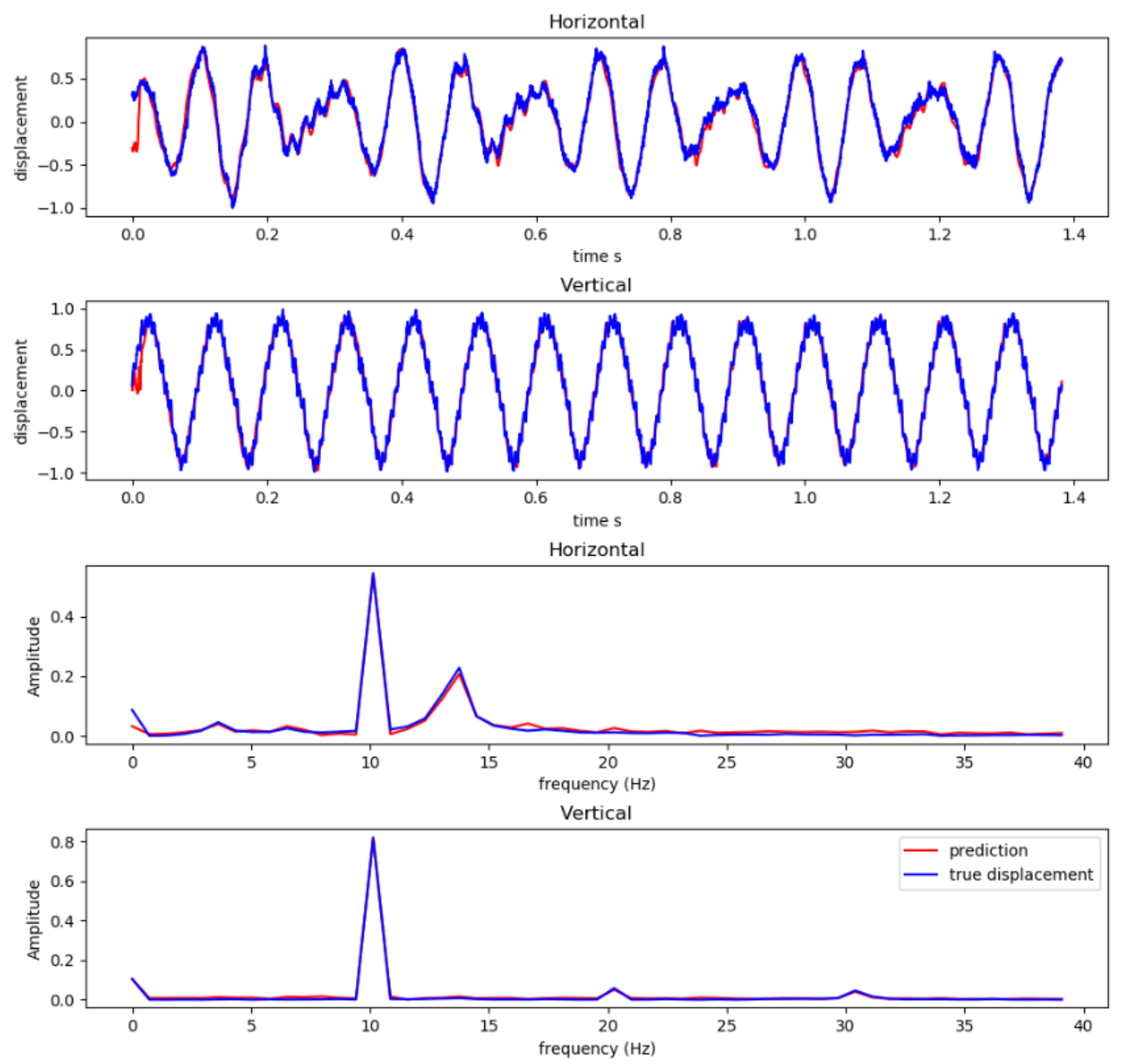

Fig. 6. Time series and spectrums of the roll center point response based on a neural network trained with measured force sensor data as input. The amplitudes have been normalized and reflect displacement units. The rotational frequency of the roll was $10.14 \mathrm{~Hz}$, MSE Loss $=0.0076$

\section{Discussion}

In this study, a recurrent neural network was successfully trained to predict center point movement of the rotor middle cross section based on measured values from the ends of the roll. The results show that the neural network can be used to predict center point movement. In this studied case, there is an obvious physical connection between the center point movement and forces or accelerations in the ends. However, it is possible for machine learning algorithms to find 
connections between variables that may seem surprising. It might also be possible to predict similar phenomena with reasonable accuracy with even less data, such as sound data.

In a larger perspective, the digital twin concept as a product-centric view to data from all of a product's lifecycle phases addresses the problem of data fragmentation into different systems. A link between the physical product and its virtual signature is needed. Although the specific technical implementation of the data transfer will vary between usecases, common features for any digital twin based systems can be identified.

The declining cost of data transfer, storage, processing and sensoring has already made possible applications not feasible in the past. Massive amounts of data and computing power will enable the leap towards a real digital twin utilizing of big data and advanced analytics to convey an accurate representation of a product's status to a human. In the context of design and production, information from fleets of digital twins can be used to improve the flow of information from operation and manufacturing back to design. When more data is collected, machine learning algorithms can be used to predict. When more data are available, inference (finding out the causes of events) might also be a possibility.

One viewpoint to the benefits of a digital twin is that the digital twin and virtual sensors are about using information as a replacement to wasted resources [10]. A digital twin system will never be able to store all data related to a product. Answering every possible question about a product would require collecting data at atom level, which is not possible now and will not be in the future. Real production chains are very complex systems and a useful digital twin will require careful consideration of what data to collect and what are the costs if the implementation and collection. To increase the benefits of such data analytics systems, the aim should be to first create virtual sensors for the most valuable identified key performance indicators in a process.

\section{Acknowledgments}

This research was funded by the Academy of Finland (TwinRotor, grant number 313675).

\section{References}

[1] Qi, Q., \& Tao, F. (2018). Digital Twin and Big Data Towards Smart Manufacturing and Industry 4.0: 360 Degree Comparison. IEEE Access, XX(c), 1-1. https://doi.org/10.1109/ACCESS.2018.2793265

[2] Tao, F., Cheng, J., Qi, Q., Zhang, M., Zhang, H., \& Sui, F. (2018). Digital twin-driven product design, manufacturing and service with big data. International Journal of Advanced Manufacturing Technology, 94(9-12), 3563-3576. https://doi.org/10.1007/s00170-017-0233-1

[3] Hribernik, Karl \& Rabe, L. \& Thoben, Klaus-Dieter \& Schumacher, Jens. (2006). The product avatar as a productinstance-centric information management concept. International Journal of Product Lifecycle Management. 1. 367 - 379. 10.1504/IJPLM.2006.011055.

[4] Haag, S., \& Anderl, R. (2018). Digital twin - Proof of concept. Manufacturing Letters, $10-12$. https://doi.org/10.1016/j.mfglet.2018.02.006

[5] Viitala, R (2018. Effect of Assembled Bearing Inner Ring Geometry on Subcritical Rotor Vibration. Doctoral dissertation, Aalto University. http://urn.fi/URN:ISBN:978-952-60-8196-0

[6] The Open Group (2018) Open Data Format (O-DF), an Open Group Internet of Things (IoT) Standard. Available from: http://www.opengroup.org/iot/odf/ Accessed: 2019-10-16

[7] Goodfellow,I., Bengio, Y., \& Courville, A. (2016). Deep Learning. MIT Press. Available from: http://www.deeplearningbook.org Accessed 2019-10-16

[8] Hochreiter, S. \& Schmidhuber, J. (1997) Long Short-Term Memory. Neural Computation, 9(8):1735-1780, 1997. https://doi.org/10.1162/neco.1997.9.8.1735

[9] Graves, A. (2013) Generating Sequences With Recurrent Neural Networks. Available from: https://arxiv.org/abs/1308.0850 Accessed 2019-10-16.

[10] Grieves, M., \& Vickers, J. (2017). Digital Twin: Mitigating Unpredictable, Undesirable Emergent Behavior in Complex Systems. In F.-J. Kahlen, S. Flumerfelt, \& A. Alves (Eds.), Transdisciplinary Perspectives on Complex Systems: New Findings and Approaches (pp. 85-113). Cham: Springer International Publishing. https://doi.org/10.1007/978-3-319-38756-7_4 